\title{
The effect of disc height on cage size in transforaminal interbody fusion (TLIF)
}

\section{Transforaminal interbody füzyonda disk yüksekliğinin kafes boyuna etkisi}

\author{
Bahadır H. GÖKÇEN ${ }^{1} \odot$, Sinan ERDOĞAN ${ }^{1} \oplus$, Erhan şÜKÜR ${ }^{2} \oplus$, Ahmet Çağrı UYAR ${ }^{2} \oplus$, Çağatay ÖZTÜRK ${ }^{1} \oplus$
}

\section{ABSTRACT}

Aim: Transforaminal interbody fusion (TLIF) technique is used to provide fusion in spine surgery. The technique itself has technical details and can lead to serious complications if not paid attention to them. Determining the size of the TLIF cage used in this process is also an important technical detail.

Method: In this retrospective study we wanted to evaluate the predictability of the cage size used in TLIF surgery. For this purpose, we examined the disc heights and cage sizes of 22 patients who underwent TLIF surgery.

Results: Nine of the patients were male, 13 were female with an overall mean age of 52.3 yeras (30-72). There was no statistically significant correlation between the sizes of the cages used, and disc heights.

Conclusion: We think that taking care of technical details is appropriate for determination of the cage size used in TLIF procedure.

Keywords: Fusion, cage, disc, height öz

Amaç: Transforaminal interbody füzyon(TLIF) tekniği omurga cerrahisinde füzyon sağlamak amacı ile kullanılmaktadır. Tekniğin kendisi teknik detaylara sahip olup, dikkat edilmemesi halinde ciddi komplikasyonlara yol açabilir. Bu işlemde kullanılan TLIF kafesinin boyunu belirlemek de teknik detayların içinde önemli bir yere sahiptir.

Yöntem: Bu retrospektif çalıșmada, TLIF cerrahisinde kullanılan kafesin önceden tahmin edilebilirliğini değerlendirmek istedik. Bu amaç ile TLIF cerrahisi uygulanmış 22 hastanın disk yüksekliklerini ve kafes boylarını inceledik.

Bulgular: Hastaların 9'u erkek, 13'ü kadın idi ve yaş ortalaması 52.3 (30-72)'tü. Kullanılan kafes boyları ve disk yükseklikleri arasında istatsiksel olarak anlamlı bir birliktelik saptanmadı.

Sonuç: Transforaminal Interbody füzyon tekniğinde kullanılan kafes boyunun cerrahi teknik detaylara dikkat ederek belirlemenin daha uygun olduğunu düşünmekteyiz.

Anahtar kelimeler: Füzyon, kafes, disk, yükseklik

\section{GiRiş}

Omurga cerrahisi ameliyatları son yıllarda giderek artış göstermektedir. Omurga cerrahisi ameliyatlarında füzyonu elde etmenin yolu eskiden posterolateral füzyondan geçse de son zamanlarda gelişen teknojinin de yardımı ile kullanılan füzyon cerahisi tekniklerinde ilerleme kaydedilmiştir. Transforaminal interbody füzyon (TLIF) tekniği de omurga cerrahisi literatüründe kabul görmüş bir füzyon cerrahisidir ${ }^{1}$. Illk olarak 1982 yılında Harms ve Rolinger tarafından posterior lomber interbody füzyonun modifikasyonu olarak tanımlanmıştır². Geleneksel posterolateral füzyona oranla daha iyi klinik sonuçlara sahip olması, posterior füzyona oranla daha fazla kaynama yüzeyi oluşturması ve spinal kordun daha az ekartasyonuna gereksinim duyulması teorik olarak avantajlarından sayılabilirler ${ }^{3-5}$. Anterior spinal kolon desteği oluş-

Received: 13.03 .2018

Accepted: 06.06.2018

${ }^{1}$ Istinye University Medical School, Orthopedics And Traumatology Department, Istanbul, Turkey

${ }^{2}$ Sakarya University Training and Research Hospital, Orthopedics and Traumatology Department, Sakarya, Turkey

Yazışma adresi: Bahadır H Gökçen, Istinye University Medical School, Orthopedics And Traumatology Department, Istanbul, Turkey

e-mail: bahadrgokcen@gmail.com

Yazarların ORCiD bilgileri:

B.H.G. 0000-0002-5374-1166, S.E. 0000-0002-8517-3925, E.Ş. 0000-0002-4697-7904, A.Ç.U. 0000-0002-8905-6320, Ç.0̈. 0000-0003-3133-206X 
turması, patolojik segmentteki lordozu daha verimli düzeltmesi, azalmış olan disk yüksekliğini onarması ve ameliyat sonrası dönemde daha sağlam stabilite oluşturması TLIF tekniğinin yıllar içinde klinik kullanımını arttırmıştır6-9. Fakat öğrenme eğrisine sahip olan TLIF prosedürü teknik detaylara dikkat edilmez ise yıkıcı komplikasyonlara yol açma potansiyeline sahiptir ${ }^{10,11}$. Özellikle TLIF prosedüründe kullanılan kafesin uygun boyda seçilmesi ve yerleştirilmesi bu tekniğin anahtar adımıdır. Cerrahi tecrübenin kafes boyuna etkisinden söz edilmiştir fakat kafesin uygun boyda olmamasının lokal disk açısında azalmaya yol açarak ameliyat sonrası prognozu etkileyebilir ${ }^{12,13}$.

Çalışmamızın amacı, TLIF prosedüründe tahmini kafes boyunun hesaplanmasında yardımcı olabilecek kriterleri belirlemektir. Bu nedenle tek seviye TLIF prosedürü uygulanan hastalarda kullanılan kafes boyunun ameliyat öncesi dönemde sağlam disk yüksekliği ve hasarlı disk yüksekliği ile olan ilişkisini değerlendirdik.

\section{GEREÇ ve YÖNTEM}

02.04.2018 tarih ve E. 4898 sayılı Etik Kurul onayı alınmıştır. 2017-2018 yılları arasında lomber omurga rahatsızlığı nedeni ile ameliyat edilen 112 hastanın bilgileri retrospektif olarak değerlendirildi. Posterolateral füzyon uygulanmış, enfeksiyon nedeni ile cerrahi geçirmiş, tümor cerrahisi ve revizyon cerrahisi uygulanmış hastalar çalışmadan çıkartıldı. Toplam 58 hastanın cerrahi kayıtları değerlendirildi. Ellisekiz hastanın 30'u birden fazla seviyeden ve L5-S1 seviyesinden TLIF cerrahisi geçirmiş idi. Geriye kalan 28 hasta tek seviye (L4-5) TLIF cerrahisi geçirmiş idi. Birden fazla seviyeden ve L5-S1 seviyesinden TLIF cerrahisi geçirmiş 30 hasta çalışmadan çıkartıldı ve tek seviye (L4-5) TLIF cerahisi geçiren 28 hasta çalışmaya dahil edildi.

Hastaların cerrahi uygulanmış disk seviyesi ile bir üst ve bir alttaki disk seviyelerinin ameliyat öncesi bilgisayarlı tomografi (BT) ve manyetik rezonans görüntülemeleri (MRG) incelendi. Proksimal ve distal disk yükseklikleri MRG'de disk dejenerasyonu açısından Pfirrmann sınıflamasıne göre sınıflandırıldı. Komşu segmentlerinde Pfirrmann evre >2 disk dejenerasyonuna sahip 6 hasta çalışmadan çıkartıldı. Geriye kalan tek seviye TLIF cerahisi geçirmiş, komşu segment disk dejenerasyonu Pfirrmann tip1 ve tip 2 olan toplam 22 hastanın yaş ve cinsiyet bilgileri kayıt edildi. TLIF kafes boyutları retrospektif olarak ameliyat notları taranarak belirlendi. Disk seviyeleri (proksimal, hastalıklı ve distal) sagittal BT kesitlerinde 3 alana bölündü (ön-orta-arka) (Figür 1). Cerrahi uygulanan seviye ve bir üst ile bir alt seviyesi olmak üzere 3 disk bölge-

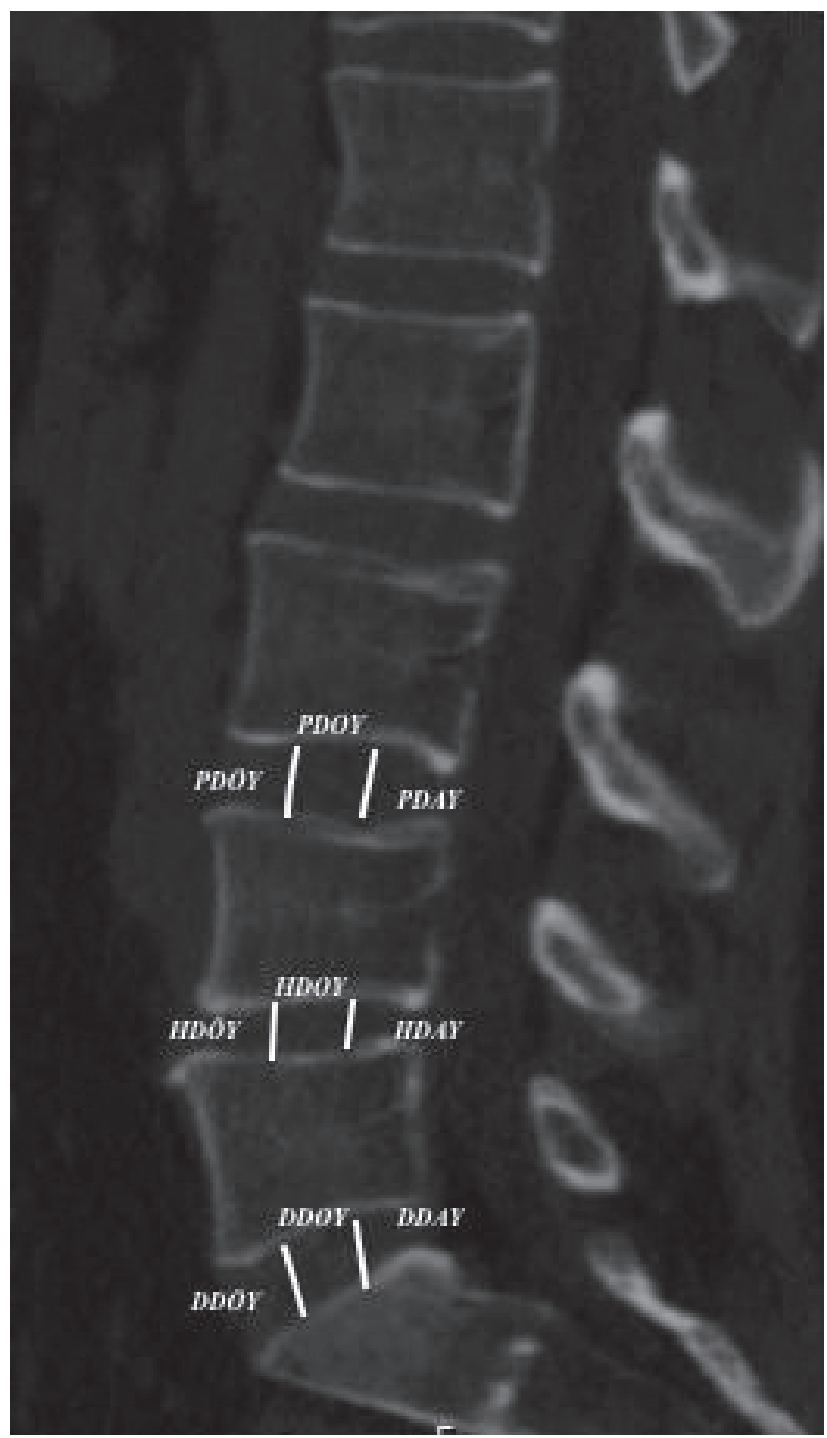

Figür 1. Sagittal Bilgisayarlı Tomografi kesitinde proksimal, hastalıklı ve distal disk yükseklikleri ölçümü. (HDÖY: Hastalıklı Disk Ön Yükseliği, HDOY: Hastalıklı disk Orta Yüksekliği, HDAY: Hastalıklı Disk Arka Yüksekliği. PDÖY: Proksimal Disk Ön Yüksekliği, PDOY: Proksimal Disk Orta Yüksekliği, PDAY: Proksimal Disk Arka Yüksekliği. DDÖY: Distal Disk Ön Yüksekliği, DDOY: Distal Disk Orta Yüksekliği, DDAY: Distal Disk Arka Yüksekliği). 
sinin ön, orta ve arka yükseklikleri bilgisayarlı tomografideki sagittal kesit görüntüleri üzerinde birisi 10 sene üzerinde deneyimli 2 omurga cerrahı tarafından ölçüldü. Ölçümler aynı kişiler tarafından bir önceki ölçümlerden bağımsız olarak 1'er ay ara ile tekrarlandı. Birinci ve ikinci ölçümdeki değerlerin kullanılan kafes boyu ile ilişkisi ve iki ölçüm arası uyum istatiksel olarak değerlendirildi.

\section{ISTATIKSEL ANALIZ}

Sürekli değişkenleri saptamak için deskriptif istatistikler değerlendirilmiştir (ortalama, standart sapma, minimum, medyan, maksimum). Bağımsız ve normal dağılan iki sürekli değişkenin karşılaştırması Student t testi ile, dağılıma uygunluk göstermeyen iki değişkenin karşılaştırması ise Mann Whitney $u$ testi ile yapılmıştır. Normal dağılan ve normal dağılmayan iki sürekli değişken arasındaki ilişkinin belirlenmesi için sırası ile Pearson korelasyon katsayısı ve Spearman's rho korelasyon katsayısı belirlenmiştir. İki ölçüm arasındaki uyum için Cronbach's Alpha katsayısı hesaplanmıştır. Bağımsız değişkenlerin sürekli bağımlı değişken üzerindeki etkisini değerlendirmek amacıyla doğrusal regresyon analizi yapılmıştır. İstatistiksel anlamlılık düzeyi 0,05 olarak belirlenmiştir. Analizler MedCalc Statistical Software version 12.7.7 (MedCalc Software bvba, Ostend, Belgium; http://www. medcalc.org; 2013) programı kullanılarak gerçekleştirilmiştir.

\section{BULGULAR}

Çalışmaya dahil edilen hastaların yaş ortalaması

Tablo 1. Cinsiyete göre kafes boyu karşılaştırması.

\begin{tabular}{lllll}
\hline & \multicolumn{1}{c}{ Erkek } & & \multicolumn{1}{c}{ Kadın } & p \\
\cline { 2 - 2 } $\begin{array}{c}\text { Ort.+SS } \\
\text { Med. (Min.-Maks.) }\end{array}$ & & $\begin{array}{c}\text { Ort.+SS } \\
\text { Med. (Min.-Maks.) }\end{array}$ \\
\hline Kafes Boyu & $10,6+0,5$ & $9,4+0,9$ & 0,003 \\
& $11(10-11)$ & $9(7-11)$ &
\end{tabular}

Cinsiyete göre kafes boyu dağılımı açısından istatistiksel anlamI farklılık vardır. (Mann-Whitney $\cup$ p<0,05). (Ort: Ortalama, SS: Standart Sapma, Med: Medyan, Min: Minimum, Max: Maksimum) $52,3^{\prime}$ tür (30-72). Yirmi iki hastanın 9'u $(\% 40,9)$ erkek, 13 'ü $(\% 59,1)$ kadındır. Cinsiyete göre kafes boyu dağılımı açısından istatistiksel anlamlı farklılık vardır $(p=0,03<0,05)$ (Tablo 1). Erkeklerin kafes boyu ortalaması 10.6, kadınların kafes boyu ortalaması 9.4'dür.

Tablo 2. Ölçümleri yapan 1. kişi ve 2. kişi arasında kusursuz düzeyde uyum vardır (Cronbach's Alpha=1,00).

\begin{tabular}{|c|c|c|c|}
\hline & 1. kişi & 2. kişi & \\
\hline & $\begin{array}{l}\text { Ort.+SS } \\
\text { Med. (Min.-Maks.) }\end{array}$ & $\begin{array}{c}\text { Ort.+SS } \\
\text { Med. (Min.-Maks.) }\end{array}$ & $\begin{array}{c}\text { Cronbach's } \\
\text { Alpha }\end{array}$ \\
\hline HDÖY & $\begin{array}{l}8,4+2,1 \\
8,1(4,2-11,9)\end{array}$ & $\begin{array}{l}8,4+2,1 \\
8,1(4,2-11,9)\end{array}$ & 1,00 \\
\hline HDOY & $\begin{array}{l}8,4+2,2 \\
8,4(2,8-12,2)\end{array}$ & $\begin{array}{l}8,4+2,2 \\
8,4(2,8-12,2)\end{array}$ & 1,00 \\
\hline HDAY & $\begin{array}{l}5,9+1,8 \\
6,1(2,0-9,1)\end{array}$ & $\begin{array}{l}5,9+1,8 \\
6,1(2,0-9,1)\end{array}$ & 1,00 \\
\hline PDÖY & $\begin{array}{l}9,06+1,9 \\
9,2(5,7-13,9)\end{array}$ & $\begin{array}{l}9,06+1,9 \\
9,2(5,7-13,9)\end{array}$ & 1,00 \\
\hline PDOY & $\begin{array}{l}10,2+1,8 \\
10,6(5,7-12,9)\end{array}$ & $\begin{array}{l}10,2+1,8 \\
10,6(5,7-12,9)\end{array}$ & 1,00 \\
\hline PDAY & $\begin{array}{l}6,9+1,7 \\
6,4(4,2-9,7)\end{array}$ & $\begin{array}{l}6,9+1,7 \\
6,4(4,2-9,7)\end{array}$ & 1,00 \\
\hline DDÖY & $\begin{array}{l}10,5+3,4 \\
11,1(3,2-15,6)\end{array}$ & $\begin{array}{l}10,5+3,4 \\
11,1(3,2-15,6)\end{array}$ & 1,00 \\
\hline DDOY & $\begin{array}{l}8,9+2,9 \\
9,2(2,3-13,3)\end{array}$ & $\begin{array}{l}8,9+2,9 \\
9,2(2,3-13,3)\end{array}$ & 1,00 \\
\hline DDAY & $\begin{array}{l}5,9+2,1 \\
5,2(1,9-9,9)\end{array}$ & $\begin{array}{l}5,9+2,1 \\
5,1(1,9-9,9)\end{array}$ & 1,00 \\
\hline
\end{tabular}

Tablo 3. Parametrelerin ölçümler arası ve kişiler arası ortalamaları.

\begin{tabular}{lll}
\hline & & Kafes Boyu \\
\hline HDÖY & $r$ & 0,145 \\
& $p$ & 0,519 \\
HDOY & $r$ & 0,228 \\
& $p$ & 0,307 \\
HDAY & $r$ & 0,246 \\
& $p$ & 0,271 \\
PDÖY & $r$ & $-0,228$ \\
& $p$ & 0,308 \\
PDOY & $r$ & $-0,093$ \\
& $p$ & 0,680 \\
PDAY & $r$ & 0,077 \\
& $p$ & 0,733 \\
DDÖY & $r$ & 0,323 \\
& $p$ & 0,143 \\
DDOY & $r$ & 0,234 \\
& $p$ & 0,295 \\
DDAY & $r$ & 0,307 \\
& $p$ & 0,165
\end{tabular}

Kafes boyu ile parametreler arasında istatistiksel anlamlı korelasyon bulunmamıştır (Spearman's rho $p=0.38>0,05$ ). 
Ölçümleri yapan iki kişinin yaptıkları ölçümler arasında ve kendi yaptıkları ölçümleri arasında kusursuz düzeyde bir uyum belirlenmiştir (Tablo 2). Bir üst seviye proksimal diski, ameliyat edilen disk seviyesi ve bir alt seviye distal diskinin kişiler arası ölçümlerinin ortalamaları alınmıştır. Kafes boyu ile parametreler arasında istatistiksel anlamlı korelasyon bulunmamıştır ( $p=0,38>0,05$ ) (Tablo 3). Bağımlı değişken kafes boyu, bağımsız değişken disk yükseklikleri olarak alındığında ameliyat edilen disk seviyesinin arka yüksekliğinde 1 birimlik değişimin kafes boyunu 0,239 birim arttırdığı görülmüştür ( $p=0,038<0,05)$ (Tablo 4). Uygulanan TLIF prosedürü esnasında ve sonrasınde işlem ile ilgili herhangi bir komplikasyona rastlanmadı.

Tablo 4. Bağımlı değişken kafes boyu.

\begin{tabular}{|c|c|c|c|c|c|}
\hline $\mathbf{R}^{2}$ & $\begin{array}{l}\text { Düzeltilmiş } \\
\mathbf{R}^{2}\end{array}$ & $\begin{array}{l}\text { Anlamiılık } \\
\text { Düzeyi p }\end{array}$ & F Değeri & & \\
\hline \multirow[t]{2}{*}{ Model 0,197 } & 0,157 & 0,038 & 4,921 & & \\
\hline & $\begin{array}{l}\text { Standardize } \\
\text { edilmemiş } \beta\end{array}$ & $\begin{array}{l}\text { Standart } \\
\text { Sapma }\end{array}$ & $\begin{array}{l}\text { Standardize } \\
\text { edilmiş } \beta\end{array}$ & $\begin{array}{l}\mathrm{t} \\
\text { değeri }\end{array}$ & $\begin{array}{l}\text { Anlamlılık } \\
\text { Düzeyi p }\end{array}$ \\
\hline Sabit & 8,444 & 0,669 & & 12,625 & $<0,001$ \\
\hline HDAY & 0,239 & 0,108 & 0,444 & 2,218 & 0,038 \\
\hline
\end{tabular}

Bağımsız değişken HDAY (hastalıklı disk arka yüksekliği) olarak alınmıştır. Model istatistiksel olarak anlamlıdır $(p=0,038<0,05)$. DAY'deki 1 br'lik değişim Kafes Boyu'nu 0,239 birim arttırmaktadır.

\section{TARTIŞMA}

Lomber füzyon cerrahisinin ana amacı sağlam bir kaynama dokusu elde etmek ve stabiliteyi sağlamaktır. İki disk arasındaki alanın biyolojik ve mekanik olarak intertransvers plandan daha üstün olduğu görüşü birçok omurga cerrahı tarafından bilinmektedir. Ayrıca disk dokusunun üstündeki ve altındaki vertebra endplatelerinin yüksek vasküler yapısı interbody füzyon cerrahisini omurga cerrahları için daha cezbedici hale getirmektedir. Fakat teorik avantajlarının yanında intertransvers yani posterolateral füzyona oranla klinik sonuçlardaki üstünlüğü de tartışma konusu olmuştur ${ }^{14-17}$. Transforaminal interbody füzyon prosedürü iki disk arasına yerleştirilen kafes ve içindeki greft dokusu ile çevresel bir füzyon alanı oluşmasını amaçlar. "Interbody füzyon" cerrahisi bir stabilizas- yon cerrahisi olsa da tek başına posterior stabilizasyon desteği olmadan uygulanması biyomekanik açıdan her yönde stabiliteyi sağlamak adına uygun bir yöntem olarak görülmez ${ }^{18}$.

Biz de kliniğimizde TLIF prosedürü uyguladığımız hastalaramıza posterior enstrumentasyon da uygulamaktayız. Uygulanan TLIF prosedürünün bir avantajı da biyomeknik olarak posterior spinal implantlara binen yükün azaltmasıdır. Literatürde $\% 56$ ile 80 oranında posteriordan konulan implantlara binen yükü azalttığı belirtilmiştir ${ }^{19}$. Transforaminal intebody füzyon prosedüründe kullanılan kafesin karakteristik yapısının da stabilizasyona ve kaynama üzerine etkisi olduğu belirtilmiştir ${ }^{20,21}$. Uygun boyda kafes yerleştirmenin disk aralığında distraksiyon uygulayarak daha iyi bir stabilizasyona olanak sağladığı biyomekanik olarak kanıtlanmışıı ${ }^{22}$. Normalden daha büyük kafes yerleştirilmesi disk aralığında fazla distraksiyona yol açarak kök gerilmesi ve nörolojik komplikasyona yol açma olasılı̆̆ını doğurur.

Lomber omurga cerrahisinin diğer ana amacı uygun lomber lordozu sağlamaktır ${ }^{23}$. Lomber lordozu sağlamak adına tanımlanmış teknikler vardır. Posteriordan uygulanan kompresyon kuvveti ile lomber lordoz sağlanabilse de arka disk yüksekliğinde azalmaya yol açarak foraminal kanal darlığına yol açabilir. Füzyonun da hedeflendiği TLIF prosedürü uygulanan hastalarda lomber lordozu sağlamada en iyi yönteminin uygun boyda kafes yerleştirilmesi olduğu belirtilmiştir ${ }^{24-26}$. Literatürde TLIF prosedüründe kullanılan kafes boyu belirlemede etkili kriterleri değerlendiren yalnızca bir çalışmaya rastladık ${ }^{12}$. Bu çalışmada, ölçümler ayakta çekilen lateral grafiler üzerinden yapılmıştır ve kafes boyunu belirlemede ameliyat edilen disk seviyesinin ön, orta ve arka yüksekliğinin etkili olduğundan söz edilmiştir. Çalışmamızda, ölçümler sagittal BT kesitleri üzerinden yapılmıştır. Ameliyat edilen seviyedeki diskin, bir üst ve bir alt diskin yüksekliğinin kullanılan kafes boyu ile ilişkisi saptanmadı fakat ameliyat edilen disk seviyesinin arka disk yüksekliğindeki artışın kafes boyundaki artış ile orantılı olduğunu saptadık. Bu da bize cerrahi tecrübenin kafes boyunu belirlemede daha etkili olabileceğini düşündürdü. Çünkü ameliyat 
esnasında TLIF prosedürünün cerrahi tekniğinde TLIF kafesini rahat yerleştirebilmek adına disk aralığının üst ve altındaki vertebra cisminin posterior çıkıntıları ince uçlu bir osteotom yardımı ile alınır ve disk aralığına rahat geçiş sağlanır. Bu şekilde endplateler daha rahat temizlenebilir. Bu işlem arka disk aralığının yüksekliğini etkiler ve kullanılacak olan kafes boyutunda değişiklik yapabilir.

Çalışmamızın eksik yönlerinden biri hasta sayımızın azlığı olarak görülebilir fakat çalışmamıza dahil ettiğimiz hasta grubumuz tek seviye TLIF işlemi yapılan ve L4-5 seviyesini içeren hastalardan oluştuğundan spesifik bir grubu içine almaktadır. Bir diğer eksik yönü ise L5-S1 seviyesini içermemesi olarak düşünülebilir fakat L5-S1 seviyesinin en alt seviye olması ve ileri dejenerasyona bağlı daralmış disk aralıklarının çalışmamızdaki ölçümleri etkileme riskinden dolayı bu seviyede TLIF uyguladığımız hastalar çalışmadan çıkartılmıştırlar. Çalışmamızın ileride çok merkezli ve daha fazla hasta sayılı çalışmalara ışık tutabileceğini düşünmekteyiz.

Sonuç olarak, TLIF prosedürü uygulanan hastalarda bazı teknik detaylara dikkat etmenin önemli olduğunu düşünmekteyiz. Cerahi sırasında endplate temizliğinin iyi yapılmasının, disk seviyesinin arka tarafının yeterli alınmasının ve TLIF prosedürü esnasında konulan kafes boyunun perioperatif kontrolünün kafes boyunu ayarlamada daha etkili olduğunu düşünmekteyiz.

\section{KAYNAKLAR}

1. Hackenberg L, Halm H, Bullmann V, et al. Transforaminal lumbar interbody fusion: a safe technique with satisfactory three to five year results. Eur Spine J. 2005;14(6):551-8. https://doi.org/10.1007/s00586-004-0830-1

2. Harms J, Rolinger H. A one-stager procedure in operative treatment of spondylolistheses: dorsal traction-reposition and anterior fusion (author's transl). Z Orthop Ihre Grenzgeb. 1982;120(3):343-7. https://doi.org/10.1055/s-2008-1051624

3. Lowe TG, Tahernia AD, O'Brien MF, et al. Unilateral transforaminal posterior lumbar interbody fusion (TLIF): indications, technique, and 2-year results. J Spinal Disord Tech. 2002;15(1):31-8. https://doi.org/10.1097/00024720-200202000-00005

4. Humphreys SC, Hodges SD, Patwardhan AG, et al. Comparison of posterior and transforaminal approaches to lumbar interbody fusion. Spine (Phila Pa 1976). 2001;26(5):567-71. https://doi.org/10.1097/00007632-200103010-00023

5. Owens RK, Carreon LY, Djurasovic M, et al. Relative benefit of TLIF versus PSF stratified by diagnostic indication. J Spinal Disord Tech. 2014;27(3):144-7. https://doi.org/10.1097/BSD.0b013e3182867470

6. Pannell WC, Savin DD, Scott TP, et al. Trends in the surgical treatment of lumbar spine disease in the United States. Spine J. 2015;15(8):1719-27. https://doi.org/10.1016/j.spinee.2013.10.014

7. Watkins RG, Hanna R, Chang D, et al. Sagittal alignment after lumbar interbody fusion: comparing anterior, lateral, and transforaminal approaches. J Spinal Disord Tech. 2013;27:253-6. https://doi.org/10.1097/BSD.0b013e31828a8447

8. Yson SC, Santos ER, Sembrano JN, et al. Segmental lumbar sagittal correction after bilateral transforaminal lumbar interbody fusion. J Neurosurg Spine. 2012;17:37-42. https://doi.org/10.3171/2012.4.SPINE111013

9. Zhou J, Wang B, Dong J, et al. Instrumented transforaminal lumbar interbody fusion with single cage for the treatment of degenerative lumbar disease. Arch Orthop Trauma Surg. 2011;131(9):1239-45. https://doi.org/10.1007/s00402-011-1292-7

10. Aoki Y, Yamagata M, Nakajima F, et al. Examining risk factors for posterior migration of fusion cages following transforaminal lumbar interbody fusion: a possible limitation of unilateral pedicle screw fixation. J Neurosurg Spine. 2010;13:381-7. https://doi.org/10.3171/2010.3.SPINE09590

11. Uzi EA, Dabby D, Tolessa E, et al. Early retropulsion of titanium- threaded cages after posterior lumbar interbody fusion: a report of two cases. Spine. 2001;26:1073-5. https://doi.org/10.1097/00007632-200105010-00017

12. Wang $\mathrm{H}$, Chen $\mathrm{W}$, Jiang J, et al. Analysis of the correlative factors in the selection of interbody fusion cage height in transforaminal lumbar interbody fusion BMC Musculoskeletal Disorders. 2016;17:9. https://doi.org/10.1186/s12891-016-0866-5

13. Hsieh PC, Koski TR, O'Shaughnessy BA, et al. Anterior lumbar interbody fusion in comparison with transforaminal lumbar interbody fusion: implications for the restoration of foraminal height, local disc angle, lumbar lordosis, and sagittal balance. J Neurosurg Spine. 2007;7:379-86. https://doi.org/10.3171/SPI-07/10/379

14. Kim KT, Lee SH, Lee YH, et al. Clinical outcomes of 3 fusion methods through the posterior approach in the lumbar spine. Spine (Phila Pa 1976). 2006;20-31(12):1351-8.

15. Fritzell $P$, Hägg $O$, Wessberg $P$, et al. Chronic low back pain and fusion: a comparison of three surgical techniques: a prospective multicenter randomized study from the Swedish lumbar spine study group. Spine (Phila Pa 1976). 2002;1;27(11):113141.

16. Jacobs WC, Vreeling A, De Kleuver M. Fusion for low-grade adult isthmic spondylolisthesis: a systematic review of the literature. Eur Spine J. 2006;15(4):391-402. https://doi.org/10.1007/s00586-005-1021-4

17. Byrd JA, Scoles PV, Winter RB, et al. Adult idiopathic scoliosis treated by anterior and posterior spinal fusion. J Bone Joint Surg Am. 1987;69(6):843-50. https://doi.org/10.2106/00004623-198769060-00008

18. Kettler A, Wilke HJ, Dietl R, et al. Stabilizing effect of posterior lumbar interbody fusion cages before and after cyclic loading. J Neurosurg. 2000;92(1):87-92.

19. Lee JY, Milne EL, Shufflebarger HL, et al. Anterior column 
support in long segment kyphosis constructs. In Proceedings of the Scoliosis Research Society 32nd Annual Meeting, St. Louis, 1997, Paper 50.

20. Brantigan JW, Steffee AD, Geiger JM. A carbon fiber implant to aid interbody lumbar fusion. Mechanical testing. Spine (Phila Pa 1976). 1991 Jun;16(6):277-82. https://doi.org/10.1097/00007632-199106001-00020

21. Kanayama M, Cunningham BW, Haggerty CJ, et al. In vitro biomechanical investigation of the stability and stressshielding effect of lumbar interbody fusion devices. J Neurosurg. 2000;93(2):259-65.

22. Goh JC, Wong HK, Thambyah A, et al. Influence of PLIF cage size on lumbar spine stability. Spine (Phila $\mathrm{Pa} 1976)$. 2000;25(1):35-40.

https://doi.org/10.1097/00007632-200001010-00008

23. Glassman SD1, Bridwell K, Dimar JR, Horton W, Berven
S, Schwab F. The impact of positive sagittal balance in adult spinal deformity.Spine (Phila Pa 1976). 2005 Sep 15;30(18):2024-9.

https://doi.org/10.1097/01.brs.0000179086.30449.96

24. Sears W. Posterior lumbar interbody fusion for lytic spondylolisthesis: restoration of sagittal balance using insert-androtate interbody spacers. Spine J. 2005;5(2):161-9.

https://doi.org/10.1016/j.spinee.2004.05.256

25. GöddeS, Fritsch E, Dienst M, et al. Influence of cage geometry on sagittal alignment in instrumented posterior lumbar interbody fusion. Spine (Phila Pa 1976). 2003;1;28(15):1693-9.

26. Brantigan JW1, Neidre A. Achievement of normal sagittal plane alignment using a wedged carbon fiber reinforced polymer fusion cage in treatment of spondylolisthesis. Spine J. 2003;3(3):186-96.

https://doi.org/10.1016/S1529-9430(02)00536-3 